\title{
Bag-valve-mask Ventilation with Airway Adjuncts Improves Neurological Outcomes of In-hospital Cardiac Arrest
}

\author{
Atsushi Yamada ${ }^{1}$, Yasuo Takeuchi ${ }^{2}$, Yuji Nishizaki ${ }^{1}$ and Hiroyuki Daida ${ }^{1}$
}

\begin{abstract}
Objective The present study was undertaken to determine the characteristics and outcome of in-hospital cardiac arrests and the effectiveness of BVM ventilation with airway adjuncts including the oropharyngeal airways and nasopharyngeal airways.

Methods Information about in-hospital cardiac arrests over a period of 6 years was retrospectively collected, and the effectiveness of BVM ventilation with airway adjuncts was analyzed using a multivariate logistic regression model.

Results During the study period, 105 (male, $n=70$; age, 68.6 \pm 14.2 years) cardiac arrests occurred, of which $95.2 \%$ developed among inpatients and $21.0 \%$ of them were witnessed. The initial rhythm of cardiac arrests was pulseless electrical activity in $63.8 \%(67 / 105)$ and respiratory failure $(44.8 \%)$ was the most common cause. Overall, a return of spontaneous circulation occurred in $76.2 \%$ of in-hospital cardiac arrests, $31.4 \%$ survived to discharge, and the neurological outcome was good (cerebral performance category-1) in $66.7 \%$ of them. Bag-valve-mask ventilation with airway adjuncts improved the neurological outcome (OR 3.52, 95\% CI $1.07,11.5)$.
\end{abstract}

Conclusion Bag-valve-mask ventilation with airway adjuncts improved neurological outcomes.

Key words: in-hospital cardiac arrest, airway adjuncts, oropharyngeal airways, nasopharyngeal airways, cerebral performance category (CPC)

(Intern Med 51: 1517-1521, 2012)

(DOI: 10.2169/internalmedicine.51.7015)

\section{Introduction}

Despite recommendations to use airway adjuncts including the oropharyngeal airways and nasopharyngeal airways during resuscitation, clinical studies have not demonstrated that these strategies improve the outcomes of in-hospital cardiac arrest.

The American Heart Association (AHA), in collaboration with the International Liaison Committee on Resuscitation (ILCOR), published the "Guidelines 2000 for Cardiopulmonary Resuscitation and Emergency Cardiovascular Care" in 2000, and coordinated "chain of survival" actions were recommended for saving life-threatening victims including cardiac arrest. Based on the guidelines, Juntendo University Hospital established an emergency care system in 2003 to cope with life-threatening conditions that occur in hospital, including sudden cardiac arrest.

We hypothesized that early and aggressive use of airway adjuncts improves neurological outcomes, because assured bag-valve-mask (BVM) ventilation with airway adjuncts provides effective and efficient oxygen supply for the brain and reduces cerebral ischemic injuries.

The present study was undertaken to assess the characteristics and outcomes of cardiac arrests that occurred at our hospital and to evaluate the effectiveness of BVM ventilation with airway adjuncts.

\section{Materials and Methods}

\section{Setting}

Juntendo University Hospital is a 1,020-bed teaching hospital comprising 35 clinical departments that attend around

${ }^{1}$ Department of Cardiology, Juntendo University, Japan and ${ }^{2}$ Department of Emergency Medicine, Teikyo University, Japan Received for publication November 27, 2011; Accepted for publication February 22, 2012

Correspondence to Dr. Atsushi Yamada, a.yamada@juntendo.ac.jp 
Table 1. Demographic Data

\begin{tabular}{|c|c|c|}
\hline Number of cardiac arrests & & 105 \\
\hline Average Age & (year \pm SD) & $68.6 \pm 14.2$ \\
\hline Gender (Male) & $(\%)$ & $67(70 / 105)$ \\
\hline Inpatiet & $(\%)$ & $95.2(100 / 105)$ \\
\hline $\begin{array}{c}\text { Time of Onset } \\
\text { (9:00 AM-8:59 PM) }\end{array}$ & (\%) & $52.4(55 / 105)$ \\
\hline Witnessed & $(\%)$ & $21.0(22 / 105)$ \\
\hline Time to ALS team arrival & (min.) & $6.4 \pm 6.0$ \\
\hline
\end{tabular}

3,900 outpatients per day. This hospital does not have the equipments to apply therapeutic hypothermia. An emergency call system [J(Juntendo)-STAT call] has been established to respond to life-threatening emergencies including cardiac arrest in our hospital. The J-STAT call simultaneously broadcasts an emergency throughout the entire hospital. Sixty individuals selected from each clinical department have been trained in basic (BLS) and advanced (ALS) life support techniques to immediately respond to J-STAT calls and promptly apply specialized interventions on site (J-STAT team). In addition, 30 automated external defibrillators (AEDs) and 34 unified emergency carts consisting of a bagvalve-mask (BVM), airway adjuncts including oropharyngeal airway (PORTEX ${ }^{\mathrm{TM}}$ Guedel Airways) and nasopharyngeal airway (PORTEX ${ }^{\mathrm{TM}}$ Nasopharyngeal airways), adrenaline (epinephrine), intubation sets and record sheets, have been installed in the outpatient department and on all floors of all wards to allow early defibrillation and emergency care on site. Cardiopulmonary resuscitation (CPR) training for other physicians and nurses at our hospital was also started in 2003.

\section{Patients and data collection}

Data regarding all cardiac arrests that occurred at our hospital and which were treated by the J-STAT team between January 1, 2003 and December 31, 2008 were retrospectively reviewed.

Data included the total number of cardiac arrests, patient age, gender and category (inpatient/outpatient), time of onset (9:00 AM to 8:59 PM or 9:00 PM to 8:59 AM), number of witnessed cardiac arrests, and time to J-STAT team arrival. An experienced cardiologist reviewed follow-up data such as the initial rhythm, causes, types of interventions, numbers of returns of spontaneous circulation (ROSC) and survival to discharge, and neurological outcomes described as cerebral performance category (CPC) values were also reviewed. Causes of cardiac arrest were retrospectively investigated and classified according to the Utstein template.

\section{Statistical analysis}

Results for continuous variables are expressed as means \pm $\mathrm{SD}$ and results for categorical variables are expressed as ratios $(\%)$. We evaluated factors influencing the prognosis of patients who went into cardiac arrest at our hospital. Data were statistically analyzed using univariate and multivariate logistic regression models to calculate odds ratios (OR) and $95 \%$ confidence intervals $(95 \% \mathrm{CI})$ in order to evaluate the relationship between neurological outcomes (CPC-1) and several factors. In logistic regression analysis, we use age, gender, yearly transition, season (April to June), time of onset (9:00 PM to 8:59 AM), witnessed, time to arrival, BVM, BVM with airway adjuncts, AED, intubation and adrenaline (epinephrine) as explanatory variables.

Statistical significance was defined as a $\mathrm{p}$ value below 0.05. All data were statistically analysed using SAS Enterprise Guide 4 software (SAS Institute Inc., Cary, NC, USA).

\section{Results}

\section{Incidence and general results}

A total of 105 (male, $n=70$; age, 68.6 \pm 14.2 years) cardiac arrests occurred at our hospital during the study period of 6 years, and inpatients accounted for $95.2 \%$ (100/105) of them. Time of onset did not significantly differ (9:00 AM to 8:59 PM vs. 9:00 PM to 8:59 AM). Among the cardiac arrests, $21.0 \%$ were witnessed. The time from recognition of cardiac arrest to the arrival of a J-STAT team was $6.4 \pm 6.0$ minutes (Table 1).

\section{Initial rhythm, causes and interventions}

The initial rhythms of cardiac arrests were ventricular fibrillation (VF)/pulseless ventricular tachycardia (pulseless VT) in $32.4 \%$ (34/105), pulseless electrical activity (PEA) in $63.8 \%(67 / 105)$ and asystole in $3.8 \%$ (4/105).

Respiratory failure was the most frequent cause, accounting for $44.8 \%(47 / 105)$, followed by hypotension in $28.6 \%$ (30/105) and myocardial infarction in $12.4 \%$ (13/105). Toxins, hypothermia, and stroke were not reported.

All victims required chest compression on site, and others required, BVM ventilation 76.2\% (80/105), BVM with Airway adjuncts $13.3 \%$ (14/105), endotracheal intubation $66.7 \%$ (70/105) and adrenaline (epinephrine) $84.8 \%$ (89/ 105). Among those with VF/pulseless VT, $3.8 \%$ (4/105) required defibrillation using an AED (Table 2).

\section{Outcome of resuscitation}

Table 3 shows the outcomes of resuscitation. A return of spontaneous circulation (ROSC) occurred in $76.2 \%$ (80/ $105), 31.4 \%$ (33/105) survived to discharge and $66.7 \%$ (22/ 33 ) experienced a good recovery (CPC-1).

According to the initial rhythm, ROSC occurred in $88.2 \%$ (30/34) of those with VF/pulseless VT, $38.2 \%$ (13/34) survived to discharge and $69.2 \%(9 / 13)$ had a good recovery (CPC-1). After initial PEA, these values were $73.1 \%$ (49/ $67), 28.4 \%(19 / 67)$ and $68.4 \%$ (13/19), respectively. The survival rate of asystole was lower than that among patients with VF/Pulseless VT and PEA, with these values being $25 \%(1 / 4), 25 \%(1 / 4)$ and $0 \%(0 / 1)$, respectively. 
Table 2. Initial Rhythm, Cause and Intervention

\begin{tabular}{cccc}
\hline Initial rhythm & VF/Pulseless VT & $(\%)$ & $32.4(34 / 105)$ \\
& PEA & $(\%)$ & $63.8(67 / 105)$ \\
Cause & Asystole & $(\%)$ & $3.8(4 / 105)$ \\
& & & \\
& Respiratory failure & $(\%)$ & $44.8(47 / 105)$ \\
& Hypotension & $(\%)$ & $28.6(30 / 105)$ \\
& Myocaridial infarction & $(\%)$ & $12.4(13 / 105)$ \\
& Hyper/Hypokalemia & $(\%)$ & $5.7(6 / 105)$ \\
& Cardiac tamponade & $(\%)$ & $1.9(2 / 105)$ \\
& Others & $(\%)$ & $6.7(7 / 105)$ \\
& & & \\
Intervention & AED & $(\%)$ & $3.8(4 / 105)$ \\
& Adrenaline & $(\%)$ & $84.8(89 / 105)$ \\
& BVM & $(\%)$ & $76.2(80 / 105)$ \\
& BVM with Airway adjuncts & $(\%)$ & $13.3(14 / 105)$ \\
& Intubation & $(\%)$ & $66.7(70 / 105)$ \\
& Oxygen & $(\%)$ & $100(105 / 105)$ \\
& Chest compression & $(\%)$ & $100(105 / 105)$ \\
\hline
\end{tabular}

\section{Neurological outcomes}

Table 4 shows the factors affecting neurological outcomes. According to neurological outcomes based on cerebral performance category (CPC) scales, univariative analysis showed that BVM ventilations with airway adjuncts including the oropharyngeal airways and nasopharyngeal airways participated good recovery (CPC-1) (OR 3.52, 95\%CI $1.07,11.5)$. Otherwise, BVM alone and endotracheal intubation did not improve neurological outcomes (OR 0.32, 95\% CI $0.12,0.84$ : OR $0.32,95 \%$ CI $0.12,0.84$ ).

Table 5 shows the results of the multivariate logistic regression analysis adjusted for age and sex.

\section{Discussion}

In-hospital cardiac arrest is a major public health problem. The key principle in resuscitation is strengthening links in the chain of survival. Successful resuscitation requires immediate recognition of cardiac arrest, activation of an emergency response system, early CPR, early defibrillation and early advanced life support. Continuous effective chest compression, attempts at early defibrillation, as well as and diagnosing and treating underlying reversible causes are important fundamental aspects of successful cardiac arrest resuscitation.

The survival rates and neurological outcomes at our hospital were better than those described by the National Registry of Cardiopulmonary Resuscitation (NRCPR) (1). Two conditions might account for this. First, the infrastructure at our hospital, including "J-STAT call" systems, 30 AEDs and 34 unified emergency carts, is very effective. Furthermore, our J-STAT teams are trained in BLS and ALS and other physicians and nurses have attended courses on resuscitation training including BLS and ALS since 2003, especially all nurses have been trained in BLS. This is important because the survival of patients with cardiac arrest requires accurate assessment of an underlying reversible cause and appropriate treatment and medical staff with the direction, coordination and integration of high quality CPR. The benefits of interventions by trained emergency medical teams on resuscitation have been reported (2-4). The nurses are the most frequent first responders in a hospital, and they immediately activated the emergency response systems at our hospital and applied BLS as capable bystanders.

Despite recommendations to use airway adjuncts including the oropharyngeal airways and nasopharyngeal airways during resuscitation, clinical studies have not demonstrated that these strategies improve the outcomes of in-hospital cardiac arrest. In this study, univariate and multivariate analysis revealed that BVM with airway adjuncts improved neurological outcomes of resuscitation at our hospital, whereas BVM alone and endotracheal intubation did not. At least two reasons might account for this finding.

First, the most frequent major initial rhythm of cardiac arrest was PEA, and the most frequent cause was respiratory failure. The application of BVM ventilation with airway adjuncts that were promptly and safely inserted by trained staff conferred better oxygenation compared with BVM alone and endotracheal intubation. Therefore cerebral ischemic injuries were reduced.

Another possible explanation for the better neurological outcomes was that nurses trained in resuscitation collaborated with the J-STAT team on site. The application of BVM ventilation is most effective when applied by two trained providers and it should never be attempted by lone providers. Airway adjuncts including oropharyngeal airways and nasopharyngeal airways are associated with several complications such as airway obstruction and bleeding, as well as inadvertent intracranial placement (5-7). Adequate training, practice and retraining are recommended for the safe use of airway adjuncts. In addition to J-STAT teams, nurses have also been trained in the use and application of airway adjuncts for BVM at our hospital.

Endotracheal intubation was once considered the optimal method of airway management during cardiac arrest. However, no prospective randomised clinical trials have yet directly compared BVM with endotracheal intubation in adult victims of cardiac arrest. In addition, Dumot et al. demonstrated that hospital inpatients who required endotracheal intubation during resuscitation had worse survival rates (8). Furthermore, endotracheal intubation has been associated with a 6-25\% incidence of unrecognised tube misplacement or displacement (9-13). Such incidences of complications are unacceptable. Therefore, BVM ventilation with airway adjuncts during resuscitation has recently been recommended. Although complications such as trauma and tube misplacement or displacement were not found in the present study, the risk of prolonged interruption of chest compression and ventilations persisted.

The present study has several limitations, the major drawback being that it is a retrospective study of a single hospital. We investigated 105 cardiac arrests, which was not adequate to evaluate prognosis compared with various types of initial pulseless rhythms. The effectiveness of airway ad- 
Table 3. Outcome of Resucitation

\begin{tabular}{cccc}
\hline & ROSC & Survival to discharge & Good recovery (CPC- 1$)$ \\
\hline Total & $76.2 \%(80 / 105)$ & $31.4 \%(33 / 105)$ & $66.7 \%(22 / 33)$ \\
VF/Pulseless VT & $88.2 \%(30 / 34)$ & $38.2 \%(13 / 34)$ & $69.2 \%(9 / 13)$ \\
PEA & $73.1 \%(49 / 67)$ & $28.4 \%(19 / 67)$ & $68.4 \%(13 / 19)$ \\
Asystole & $25 \%(1 / 4)$ & $25 \%(1 / 4)$ & $0 \%(0 / 1)$ \\
\hline
\end{tabular}

Table 4. Factors Affecting Neurological Outcomes

\begin{tabular}{cccc}
\hline & OR & $95 \%$ CI & p value \\
\hline Good recovery (CPC-1) & & Univariate analysis & \\
\hline Age & 0.99 & $0.97,1.03$ & 0.92 \\
Gender (Male) & 2.68 & $0.83,8.65$ & 0.10 \\
Transition (1 years) & 1.12 & $0.82,1.55$ & 0.47 \\
Season & 1.01 & $0.30,3.41$ & 0.99 \\
(April - June) & & & 0.10 \\
Hour of day & 0.43 & $0.16,1.17$ & 0.41 \\
(9:00 PM - 8:59 AM) & & & 0.64 \\
Witnessed & 1.57 & $0.53,4.65$ & 0.06 \\
Time to arrival & 0.98 & $0.90,1.07$ & 0.89 \\
Airway adjuncts & 3.08 & $0.96,9.90$ & $0.04^{*}$ \\
BVM & 1.08 & $0.35,3.30$ & 0.60 \\
BVM with Airway adjuncts & 3.52 & $1.07,11.5$ & $0.02^{*}$ \\
AED & 1.93 & $0.17,22.3$ & 0.06 \\
Intubation & 0.32 & $0.12,0.84$ & \multirow{2}{*}{ means $\mathrm{p}<0.05$} \\
Adrenaline & 0.32 & $0.10,1.04$ &
\end{tabular}

Table 5. Effectiveness of Airway Adjuncts

\begin{tabular}{|c|c|c|c|}
\hline & $\mathrm{OR}$ & $95 \%$ CI & $\mathrm{p}$ value \\
\hline Good recovery (CPC-1) & \multicolumn{3}{|c|}{$\begin{array}{c}\text { Multivariate analysis } \\
\text { (adjusted for age and sex }\end{array}$} \\
\hline BVM with Airway adjuncts & 3.50 & $1.04,11.8$ & $0.04^{*}$ \\
\hline Intubation & 0.34 & $0.13,0.91$ & $0.03^{*}$ \\
\hline
\end{tabular}

juncts was suggested, but only for 14 patients. Furthermore, we did not evaluate the quality of resuscitations on site and this is important because chest compression rates, compression depth and hand-off time affect the prognosis of patients with cardiac arrest who undergo resuscitation. Although nurses trained in BLS and J-STAT teams trained in ALS applied resuscitation on site, the retrospective verification of quality is impossible. In addition, to verify the prognosis accurately, further investigation regarding patient background, medical history and complications are needed.

To further improve survival rates and neurological outcomes after resuscitation at our hospital, we will implement several reorganisations based on the features and settings of cardiac arrests revealed by this study. First, the numbers of endotracheal intubations should be reduced and the use of airway adjuncts should be increased. Indeed, the most common cause of cardiac arrest at our hospital was respiratory failure. However, the rate of endotracheal intubation was much higher than predicted.

Secondly, arranging resuscitation training programs based on the results of this study should cultivate good capabilities of assessment, judgment and clinical skills for resuscitation, and the ability to adjust for needs on site.

Thirdly, a multi-faceted approach is needed to bring about improvements. The International Liaison Committee on Resuscitation (ILCOR) has recommended evaluation of the quality and effectiveness of life-saving activities by hospital committees since 1997. Regular committee meetings will be held regularly at our hospital, various directions of improvement measures will be determined on a timely basis, and feedback will also be directly provided to all staff members.

\section{Conclusions}

BVM ventilation with airway adjuncts including the oropharyngeal and nasopharyngeal airways improved neurological outcomes after in-hospital cardiac arrest. Further improvements in survival rates and neurological outcomes after resuscitation, as well as feedback about clinical scenes based on actual situation data are needed.

The authors state that they have no Conflict of Interest (COI).

\section{References}

1. Peberdy MA, Kaye W, Ornato JP, et al. NRCPR Investigators. Cardiopulmonary resuscitation of adults in the hospital: a report of 14720 cardiac arrests from the National Registry of Cardiopulmonary Resuscitation. Resuscitation 58: 297-308, 2003.

2. Buist MD, Moore GE, Bernard SA, et al. Effects of a medical emergency team on reduction of incidence of and mortality from unexpected cardiac arrests in hospital: preliminary study. BMJ 324: 387-390, 2002.

3. Jones D, Bellomo R, Bates S, et al. Long term effect of a medical emergency team on cardiac arrests in a teaching hospital. Critical Care 9: R808-R815, 2005.

4. Konrad D, Jäderling G, Bell M, et al. Reducing in-hospital cardiac arrests and hospital mortality by introducing a medical emergency team. Intensive Care Med 36: 100-106, 2009.

5. Stonehan MD. The nasopharyngeal airway. Assessment of position by fibreoptic laryngoscopy. Anaesthesia 48: 575-580, 1993.

6. Schade K, Borzotta A, Michaels A. Intracranial malposition of nasopharyngeal airway. J Trauma 49: 967-968, 2000.

7. Muzzi DA, Losasso TI, Cuchiara RF. Complication from a nasopharyngeal airway in a patient with a basilar skull fracture. Anesthesiology 74: 366-368, 1991.

8. Dumot JA, Burval DJ, Sprug J, et al. Outcome of adult cardiopulmonary resuscitation at tertiary referral center including results of 
"limited” resuscitation. Arch Intern Med 161: 1751-1758, 2001.

9. Jones JH, Murphy MP, Dickson RL, Somerville GG, Brizendine EJ. Emergency physician-verified out-of-hospital intubation: miss rates by paramedics. Acad Emerg Med 11: 707-709, 2004.

10. Sayre MR, Sakles JC, Mistler AF, Evance JL, Kramer AT, Panioli AM. Field trial of endotracheal intubation by basic EMTs. Ann Emerg Med 31: 228-233, 1998.

11. Katz SH, Falk JL. Misplaced endotracheal tubes by paramedics in an urban emergency medical services system. Am Emerg Med 37: 32-37, 2001.
12. Jemmett ME, Kendal KM, Fourre MW, Burton JH. Unrecognized misplacement of endotracheal tubes in a mixed urban to rural emergency medical services setting. Acad Emerg Med 10: 961965, 2003.

13. Silvestri S, Ralls GA, Thundiyil J, et al. The effectiveness of outof-hospital use of continuous end-tidal carbon dioxide monitoring on the rate of unrecognized misplacement intubation within a regional emergency medical services system. Ann Emerg Med 45: 497-503, 2005.

\footnotetext{
(C) 2012 The Japanese Society of Internal Medicine http://www.naika.or.jp/imindex.html
} 